PROCEEDINGS OF THE

AMERICAN MATHEMATICAL SOCIETY

Volume 138, Number 3, March 2010, Pages 929-936

S 0002-9939(09)10046-1

Article electronically published on October 28, 2009

\title{
CHAOS FOR COWEN-DOUGLAS OPERATORS
}

\author{
BINGZHE HOU, PUYU CUI, AND YANG CAO \\ (Communicated by Bryna Kra)
}

\begin{abstract}
In this article, we provide a sufficient condition which gives Devaney chaos and distributional chaos for Cowen-Douglas operators. In fact, we obtain a distributionally chaotic criterion for bounded linear operators on Banach spaces.
\end{abstract}

\section{INTRODUCTION AND PRELIMINARIES}

A discrete dynamical system is simply a continuous mapping $f: X \rightarrow X$ where $X$ is a complete separable metric space. For $x \in X$, the orbit of $x$ under $f$ is $\operatorname{Orb}(f, x)=\left\{x, f(x), f^{2}(x), \ldots\right\}$, where $f^{n}=f \circ f \circ \cdots \circ f$ is the $n$th iteration of $f$.

Recall that $f$ is transitive if for any two nonempty open sets $U$ and $V$ in $X$, there exists an integer $n \geq 1$ such that $f^{n}(U) \cap V \neq \phi$. It is well known that, in a complete metric space without isolated points, transitivity is equivalent to the existence of dense orbit ([15]). $f$ is weakly mixing if $(f \times f, X \times X)$ is transitive. $f$ is strongly mixing if for any two nonempty open sets $U, V$ in $X$, there exists an integer $m \geq 1$ such that $f^{n}(U) \cap V \neq \phi$ for every $n \geq m$. $f$ has sensitive dependence on initial conditions (or simply $f$ is sensitive) if there is a constant $\delta>0$ such that for any $x \in X$ and any neighborhood $U$ of $x$, there exists a point $y \in X$ such that $d\left(f^{n}(x), f^{n}(y)\right)>\delta$, where $d$ denotes the metric on $X$.

In 1975, Li and Yorke 9 observed complicated dynamical behavior for the class of interval maps with period 3. This phenomena is currently known under the name of Li-Yorke chaos. Therefrom, several kinds of chaos were well studied. In the present article, we focus on Devaney chaos and distributional chaos.

Following Devaney [3],

Definition 1.1. Let $(X, f)$ be a dynamical system. $f$ is chaotic if

$(D 1) f$ is transitive;

$(D 2)$ the periodic points for $f$ are dense in $X$; and

(D3) $f$ has sensitive dependence on initial conditions.

It was shown by Banks et al. [1] that $(D 1)+(D 2)$ implies $(D 3)$ for any aperiodic system. Devaney chaos is a stronger version of chaos than Li-Yorke chaos, which was given by Huang and Ye [8] and Mai [12].

Received by the editors January 4, 2009, and, in revised form, April 16, 2009.

2000 Mathematics Subject Classification. Primary 47B37, 47B99; Secondary 54H20, 37B99.

Key words and phrases. Cowen-Douglas operators, strongly mixing, Devaney chaos, distributional chaos.

The first author is supported by the Youth Foundation of the Department of Mathematics, Jilin University. 
From Schweizer and Smítal's paper [14, distributional chaos is defined in the following way. For any pair $\{x, y\} \subset X$ and any $n \in \mathbb{N}$, define distributional function $F_{x y}^{n}: \mathbb{R} \rightarrow[0,1]$

$$
F_{x y}^{n}(\tau)=\frac{1}{n} \#\left\{0 \leq i \leq n-1 ; d\left(f^{i}(x), f^{i}(y)\right)<\tau\right\} .
$$

Furthermore, define

$$
\begin{aligned}
& F_{x y}(\tau)=\liminf _{n \rightarrow \infty} F_{x y}^{n}(\tau), \\
& F_{x y}^{*}(\tau)=\limsup _{n \rightarrow \infty} F_{x y}^{n}(\tau) .
\end{aligned}
$$

Both $F_{x y}$ and $F_{x y}^{*}$ are nondecreasing functions and may be viewed as cumulative probability distributional functions satisfying $F_{x y}(\tau)=F_{x y}^{*}(\tau)=0$ for $\tau<0$.

Definition 1.2. $\{x, y\} \subset X$ is said to be a distributionally chaotic pair if

$$
F_{x y}^{*}(\tau) \equiv 1, \quad \forall \tau>0 \quad \text { and } \quad \exists \epsilon>0, \quad F_{x y}(\epsilon)=0 .
$$

Furthermore, $f$ is called distributionally chaotic if there exists an uncountable subset $D \subseteq X$ such that each pair of two distinct points is a distributionally chaotic pair. Moreover, $D$ is called a distributionally $\epsilon$-scrambled set.

Distributional chaos always implies Li-Yorke chaos, as it requires more complicated statistical dependence between orbits than the existence of points which are proximal but not asymptotic. The converse implication is not true in general. However, in practice, even in the simple case of Li-Yorke chaos, it might be quite difficult to prove chaotic behavior from the very definition. Such attempts have been made in the context of linear operators (see [4, 5]). Further results of [4] were extended in [13. to distributional chaos for the annihilation operator of a quantum harmonic oscillator. For more about distributional chaos, one can see [16, 17, 10, 11, 18.

Let $\mathcal{H}$ be a complex separable Hilbert space, and let $\mathcal{L}(\mathcal{H})$ denote the collection of bounded linear operators on $\mathcal{H}$. We are interested in a family of operators given by Cowen and Douglas [2].

Definition 1.3. For $\Omega$ a connected open subset of $\mathbb{C}$ and $n$ a positive integer, let $\mathcal{B}_{n}(\Omega)$ denote the operators $T$ in $\mathcal{L}(\mathcal{H})$ which satisfy

(a) $\Omega \subseteq \sigma(T)=\{\omega \in \mathbb{C}: T-\omega$ not invertible;

(b) $\operatorname{ran}(T-\omega)=\mathcal{H}$ for $\omega$ in $\Omega$;

(c) $\bigvee \operatorname{ker}_{\omega \in \Omega}(T-\omega)=\mathcal{H}$; and

(d) $\operatorname{dim} \operatorname{ker}(T-\omega)=n$ for $\omega$ in $\Omega$.

We have known some properties of these operators from [2].

Proposition 1.4. Let $T \in \mathcal{B}_{n}(\Omega)$ and $\omega_{0} \in \Omega$. Then $\bigvee_{k=1}^{\infty} \operatorname{ker}\left(T-\omega_{0}\right)^{k}=\mathcal{H}$.

Proposition 1.5. If $\Omega_{0} \subseteq \Omega$ is a bounded connected open subset of $\mathbb{C}$, then $B_{n}(\Omega) \subseteq B_{n}\left(\Omega_{0}\right)$.

In this paper, $S$ is always used to denote the unit circle in $\mathbb{C}$. In the next section, we provide a sufficient condition, $\Omega \cap S \neq \phi$, which gives Devaney chaos for Cowen-Douglas operators. In the last section, we obtain a distributionally chaotic criterion for bounded linear operators on Banach spaces. By this distributionally chaotic criterion, $\Omega \cap S \neq \phi$ is also a sufficient condition which gives distributional chaos for Cowen-Douglas operators. 


\section{Devaney ChaOs For Cowen-Douglas operators}

Proposition 2.1. Let $T \in \mathcal{B}_{n}(\Omega)$. If $\Omega \cap S \neq \phi$, then $T$ is strongly mixing.

Proof. Suppose $U$ and $V$ are arbitrary open subsets in $\mathcal{H}$. We have $\epsilon>0$ and open subsets $U^{\prime}$ and $V^{\prime}$ such that

$$
B\left(u^{\prime}, \epsilon\right) \subseteq U \text { and } B\left(v^{\prime}, \epsilon\right) \subseteq V,
$$

for any $u^{\prime} \in U^{\prime}$ and any $v^{\prime} \in V^{\prime}$. Since $\Omega$ is a connected open subset and $\Omega \cap S \neq \phi$, there are two bounded connected open subsets $\Omega_{1}$ and $\Omega_{2}$ in $\Omega$ such that

$$
\sup _{\alpha \in \Omega_{1}}|\alpha|=\lambda<1 \text { and } \inf _{\beta \in \Omega_{2}}|\beta|=\rho>1 \text {. }
$$

By Proposition 1.5. there exist two points $x \in U^{\prime}$ and $y \in V^{\prime}$ with the following forms:

$$
x=\sum_{i=1}^{t} x_{i} \text { and } y=\sum_{j=1}^{l} y_{j}
$$

where $x_{i} \in \operatorname{ker}\left(T-\lambda_{i}\right), \lambda_{i} \in \Omega_{1}$, and $y_{j} \in \operatorname{ker}\left(T-\rho_{j}\right), \rho_{j} \in \Omega_{2}$.

Now let $M=\max \left\{\sum_{i=1}^{t}\left\|x_{i}\right\|, \sum_{j=1}^{l}\left\|y_{j}\right\|\right\}$. Then there is a positive integer $N$ such that for each $k \geq N$,

$$
\lambda^{k}<\epsilon / M \quad \text { and } \quad \rho^{-k}<\epsilon / M .
$$

Given any $k \geq N$, let $u(k)=x+\sum_{j=1}^{l} \rho_{j}^{-k} y_{j}$. Obviously,

$$
\|u(k)-x\|=\left\|\sum_{j=1}^{l} \rho_{j}^{-k} y_{j}\right\| \leq \sum_{j=1}^{l}\left|\rho_{j}^{-k}\right| \cdot\left\|y_{j}\right\| \leq \rho^{-k} \sum_{j=1}^{l}\left\|y_{j}\right\|<\epsilon,
$$

so $u(k) \in U$. On the other hand,

$$
\left\|T^{k} u(k)-y\right\|=\left\|\sum_{i=1}^{t} \lambda_{i}^{k} x_{i}\right\| \leq \sum_{i=1}^{t}\left|\lambda_{i}^{k}\right| \cdot\left\|x_{i}\right\| \leq \lambda^{k} \sum_{i=1}^{t}\left\|x_{i}\right\|<\epsilon,
$$

which implies $T^{k} u(k) \in V$. Hence, $T^{k}(U) \cap V \neq \phi$ and consequently $T$ is strongly mixing.

Proposition 2.2. Let $T \in \mathcal{B}_{n}(\Omega)$. If $\Omega \cap S \neq \phi$, then $\operatorname{Per}(T)$ is dense in $\mathcal{H}$.

Proof. Let $\Delta=\left\{e^{2 \pi r i}\right.$ : for all rational numbers $\left.r\right\}$. Then $\Delta$ is dense in $S$, and one can see that for each $\delta \in \Delta$, there exists a positive integer $m(\delta)$ such that $\delta$ is a root of the equation $z^{m(\delta)}=1$. Since $\Omega$ is a connected open subset and $\Omega \cap S \neq \phi$, we have $\Omega \cap \Delta \neq \phi$. Now let $s \in \Omega \cap \Delta$. Given any $k \in \mathbb{N}$. If $x \in \operatorname{ker}(T-s)^{k}$, then

$$
\begin{aligned}
T^{2 k m(s)}(x)-x & =\left(T^{2 k m(s)}-s^{2 k m(s)}\right) x \\
& =\left(T^{k m(s)}+s^{k m(s)}\right)\left(T^{k m(s)}-s^{k m(s)}\right) x \\
& =\left(T^{k m(s)}+s^{k m(s)}\right) 0=0 .
\end{aligned}
$$

Thus, $x \in \operatorname{Per}(T)$ and hence $\bigcup_{k=1}^{\infty} \operatorname{ker}(T-s)^{k} \subseteq \operatorname{Per}(T)$. Notice that if $x, y \in$ $\operatorname{Per}(T)$, then $x+y \in \operatorname{Per}(T)$. Therefore, by Proposition 1.4 $\operatorname{Per}(T)$ is dense in $\mathcal{H}$.

By Propositions 2.1 and 2.2 . one can see the following result immediately.

Theorem 2.3. Let $T \in \mathcal{B}_{n}(\Omega)$. If $\Omega \cap S \neq \phi$, then $T$ is Devaney chaotic. 
Remark 2.4. Notice that $\Omega \cap S \neq \phi$ is not a necessary condition for Devaney chaos for Cowen-Douglas operators. As is well known, the backward shift operator $T$, with the weight sequence $\left\{\omega_{n}=\frac{n+1}{n}\right\}_{n=1}^{\infty}$, is a Devaney chaotic Cowen-Douglas operator. However, the largest connected open domain $\Omega$ for $T$ which admits $T \in B_{1}(\Omega)$ is the unit open disk and hence is disjoint with $S$.

\section{Distributionally ChaOtic Criterion AND ITS APPLICATIONS TO COWEN-DOUGLAS OPERATORS}

First of all, we will give a new concept which is very useful in proving that a bounded linear operator is distributional chaotic.

Definition 3.1. Let $X$ be a Banach space and let $T \in \mathcal{L}(X)$. $T$ is called a special operator if we have a constant $\gamma>1$ such that for any $m \in \mathbb{N}$, there exists a nontrivial point $x_{m} \in X$ satisfying

(NU1) $\quad \lim _{k \rightarrow \infty}\left\|T^{k} x_{m}\right\|=0$,

(NU2) $\quad\left\|T^{i} x_{m}\right\| \geq \gamma^{i}\left\|x_{m}\right\|, \quad i=1,2, \ldots, m$.

Furthermore, such $\gamma$ is said to be a special constant for the special operator $T$.

Remark 3.2. If $x$ is the point referred to in the above definition, then for any $c \in \mathbb{C}$, $c x$ has the same properties as $x$ because of the linearity of $T$. Therefore, we can select a point with arbitrary nonzero norm satisfying the same conditions.

Theorem 3.3 (Distributionally Chaotic Criterion). Let $X$ be a Banach space and let $T \in \mathcal{L}(X)$. If $T$ is special, then $T$ is distributionally chaotic.

Proof. Let $R=\|T\|$ and let $\gamma$ be a special constant for $T$. Suppose $\left\{\epsilon_{k}\right\}_{k=1}^{\infty}$ is a sequence of positive numbers decreasing to zero. First of all, fix $N_{1} \in \mathbb{N}$ (for example, set $N_{1}=2$ ). Then there is $x_{1}$ such that $\left\|x_{1}\right\|=1$ and

$$
\lim _{k \rightarrow \infty}\left\|T^{k} x_{1}\right\|=0 \text { and }\left\|T^{i} x_{1}\right\| \geq \gamma^{i}\left\|x_{1}\right\|, \quad i=1, \ldots, N_{1} .
$$

So we can choose a positive integer $M_{1}$ such that $\left\|T^{n} x\right\|<\epsilon_{1}$ for any $n \geq M_{1}$. For convenience, let $N_{1}^{\prime}=0$. Then $\left\|T^{i} x_{1}\right\| \geq 1, \quad i=N_{1}^{\prime}, \ldots, N_{1}$.

Now we will construct a sequence of points $\left\{x_{k}\right\}_{k=1}^{\infty}$ associated with three sequences of integers $\left\{N_{k}\right\}_{k=1}^{\infty},\left\{N_{k}^{\prime}\right\}_{k=1}^{\infty}$, and $\left\{M_{k}\right\}_{k=1}^{\infty}$ such that for every $k \geq 2$,

(I) $\left\|x_{k}\right\|=R^{-M_{k-1}} \cdot 2^{-k} \cdot \epsilon_{k-1}$;

(II) $\left\|T^{i} x_{k}\right\| \geq \gamma^{i}\left\|x_{k}\right\|, \quad i=1, \ldots, N_{k}$;

(III) $\gamma^{N_{k}^{\prime}} \cdot R^{-M_{k-1}} \cdot 2^{-k} \cdot \epsilon_{k-1}>1$;

(IV) $\frac{N_{k}-N_{k}^{\prime}}{N_{k}}>\frac{k-1}{k}$;

(V) $\sum_{j=1}^{k}\left\|T^{n} x_{j}\right\|<\epsilon_{k}$, for any $n \geq M_{k}$.

Select $N_{2}^{\prime} \in \mathbb{N}$ with $\gamma^{N_{2}^{\prime}} \cdot R^{-M_{1}} \cdot 2^{-2} \cdot \epsilon_{1}>1$. Consequently, we have $N_{2} \in \mathbb{N}$ such that $\frac{N_{2}-N_{2}^{\prime}}{N_{2}}>\frac{2-1}{2}=\frac{1}{2}$. And then there is $x_{2}$ such that $\left\|x_{2}\right\|=R^{-M_{1}} \cdot 2^{-2} \cdot \epsilon_{1}$ and

$$
\lim _{k \rightarrow \infty}\left\|T^{k} x_{2}\right\|=0 \quad \text { and }\left\|T^{i} x_{2}\right\| \geq \gamma^{i}\left\|x_{2}\right\|, \quad i=1, \ldots, N_{2} .
$$

So we can choose $M_{2}$ such that $\left\|T^{n} x_{1}\right\|+\left\|T^{n} x_{2}\right\|<\epsilon_{2}$ for any $n \geq M_{2}$.

Continue in this manner. If we have obtained $\left\{x_{k}\right\}_{k=1}^{m},\left\{N_{k}\right\}_{k=1}^{m},\left\{N_{k}^{\prime}\right\}_{k=1}^{m}$, and $\left\{M_{k}\right\}_{k=1}^{m}$ such that for each $k=2, \ldots, m$,

(1) $\left\|x_{k}\right\|=R^{-M_{k-1}} \cdot 2^{-k} \cdot \epsilon_{k-1}$;

(2) $\left\|T^{i} x_{k}\right\| \geq \gamma^{i}\left\|x_{k}\right\|, \quad i=1, \ldots, N_{k}$;

(3) $\gamma^{N_{k}^{\prime}} \cdot R^{-M_{k-1}} \cdot 2^{-k} \cdot \epsilon_{k-1}>1$; 
(4) $\frac{N_{k}-N_{k}^{\prime}}{N_{k}}>\frac{k-1}{k}$;

(5) $\sum_{j=1}^{k}\left\|T^{n} x_{j}\right\|<\epsilon_{k}$, for any $n \geq M_{k}$,

then select $N_{m+1}^{\prime} \in \mathbb{N}$ with $\gamma^{N_{m+1}^{\prime}} \cdot R^{-M_{m}} \cdot 2^{-(m+1)} \cdot \epsilon_{m}>1$. Consequently, we have $N_{m+1} \in \mathbb{N}$ such that $\frac{N_{m+1}-N_{m+1}^{\prime}}{N_{m+1}}>\frac{m+1-1}{m+1}=\frac{m}{m+1}$. And then there is $x_{m+1}$ such that $\left\|x_{m+1}\right\|=R^{-M_{m}} \cdot 2^{-(m+1)} \cdot \epsilon_{m}$ and

$$
\lim _{k \rightarrow \infty}\left\|T^{k} x_{m+1}\right\|=0, \quad \text { and } \quad\left\|T^{i} x_{m+1}\right\| \geq \gamma^{i}\left\|x_{m+1}\right\|, \quad i=1, \ldots, N_{m+1} .
$$

So we can choose $M_{m+1}$ such that $\sum_{j=1}^{m+1}\left\|T^{n} x_{j}\right\|<\epsilon_{m+1}$ for any $n \geq M_{m+1}$.

Therefore, we obtain a sequence of points $\left\{x_{k}\right\}_{k=1}^{\infty}$ associated with three sequences of integers $\left\{N_{k}\right\}_{k=1}^{\infty},\left\{N_{k}^{\prime}\right\}_{k=1}^{\infty}$, and $\left\{M_{k}\right\}_{k=1}^{\infty}$ satisfying conditions (I-V). Moreover, conditions (I-III) imply the following statements:

(VI) $\sum_{k=1}^{\infty}\left\|x_{k}\right\|$ is finite.

(VII) For each $p,\left\|T^{i} x_{k}\right\|<2^{-k} \epsilon_{k-1}$, for any $k>p$ and any $1 \leq i \leq M_{p}$. Hence, $\sum_{k=p+1}^{\infty}\left\|T^{i} x_{k}\right\|<\sum_{k=p+1}^{\infty} 2^{-k} \epsilon_{k-1}<\epsilon_{p}$ for any $1 \leq i \leq M_{p}$.

(VIII) For each $k,\left\|T^{i} x_{k}\right\| \geq 1, i=N_{k}^{\prime}, \ldots, N_{k}$.

Notice that $M_{k}>N_{k}>N_{k}^{\prime}>M_{k-1}$ for each $k$ by the manner of our construction. Then we have

$\left(\mathrm{V}^{\prime}\right) \sum_{j=1}^{k-1}\left\|T^{n} x_{j}\right\|<\epsilon_{k-1}$, for $n=N_{k}^{\prime}, \ldots, N_{k}$.

$\left(\mathrm{VII}^{\prime}\right)$ For each $p, \sum_{k=p+1}^{\infty}\left\|T^{n} x_{k}\right\|<\epsilon_{p}, n=N_{p}^{\prime}, \ldots, N_{p}$.

Let $\Sigma_{2}=\{0,1\}^{\mathbb{N}}$ be a symbolic space with two symbols. According to condition (VI), we can define a map $f: \Sigma_{2} \rightarrow X$ as

$$
f(\xi)=\sum_{k=1}^{\infty} \xi_{k} x_{k}
$$

for every element $\xi=\left(\xi_{1}, \xi_{2}, \ldots\right) \in \Sigma_{2}$.

Obviously one can get an uncountable subset $D \in \Sigma_{2}$ such that for any two distinct points $\xi, \xi^{\prime} \in D, \xi$ and $\xi^{\prime}$ have infinite coordinates that are different and infinite coordinates that are equivalent. Then

$$
d\left(f(\xi), f\left(\xi^{\prime}\right)\right)=\|f(\xi)-f(\xi)\|=\left\|\sum_{k=1}^{\infty}\left(\xi_{k}-\xi_{k}^{\prime}\right) x_{k}\right\| .
$$

Set $\theta=\left(\theta_{1}, \theta_{2}, \ldots\right)=\left(\xi_{1}-\xi_{1}^{\prime}, \xi_{2}-\xi_{2}^{\prime}, \ldots\right)$. Then $d\left(f(\xi), f\left(\xi^{\prime}\right)\right)=\left\|\sum_{k=1}^{\infty} \theta_{k} x_{k}\right\|$. Note that the possible values of $\xi_{k}-\xi_{k}^{\prime}$ are only $0,-1$, or 1 , and $\theta$ has infinite coordinates being zero and infinite coordinates being nonzero.

Now we will prove that $\left\{f(\xi), f\left(\xi^{\prime}\right)\right\}$ is a distributionally chaotic pair.

Let $z=\sum_{k=1}^{\infty} \theta_{k} x_{k}$. Suppose $\left\{k_{q}\right\}_{q=1}^{\infty}$ is the infinite subsequence such that the $k_{q}$ th coordinate of $\theta$ is nonzero $(1$ or -1$)$ and $\left\{k_{r}\right\}_{r=1}^{\infty}$ is the infinite subsequence such that the $k_{r}$ th coordinate of $\theta$ is zero.

By $\left(\mathrm{V}^{\prime}\right),\left(\mathrm{VII}^{\prime}\right)$ and (VIII), for $n=N_{k_{q}}^{\prime}, \ldots, N_{k_{q}}$,

$$
\left\|T^{n} z\right\| \geq\left\|T^{n}\left(\theta_{k_{q}} x_{k_{q}}\right)\right\|-\sum_{j=1}^{k_{q}-1}\left\|T^{n} x_{j}\right\|-\sum_{j=k_{q}+1}^{\infty}\left\|T^{n} x_{j}\right\|>1-\epsilon_{k_{q}-1}-\epsilon_{k_{q}} .
$$


Since $\left\{\epsilon_{k}\right\}_{k=1}^{\infty}$ decreases to zero,

$$
\begin{aligned}
F_{f(\xi) f\left(\xi^{\prime}\right)}\left(\frac{1}{2}\right) & =\liminf _{n \rightarrow \infty} F_{f(\xi) f\left(\xi^{\prime}\right)}^{n}\left(\frac{1}{2}\right) \\
& \leq \liminf _{q \rightarrow \infty} F_{f(\xi) f\left(\xi^{\prime}\right)}^{N_{k_{q}}}\left(\frac{1}{2}\right) \\
& \leq \lim _{q \rightarrow \infty} \frac{N_{k_{q}}^{\prime}}{N_{k_{q}}} \\
& \leq \lim _{q \rightarrow \infty} \frac{1}{k_{q}}=0 .
\end{aligned}
$$

On the other hand, for $n=N_{k_{r}}^{\prime}, \ldots, N_{k_{r}}$,

$$
\left\|T^{n} z\right\| \leq\left\|T^{n}\left(\theta_{k_{r}} x_{k_{r}}\right)\right\|+\sum_{j=1}^{k_{r}-1}\left\|T^{n} x_{j}\right\|+\sum_{j=k_{r}+1}^{\infty}\left\|T^{n} x_{j}\right\| \leq \epsilon_{k_{r}-1}+\epsilon_{k_{r}} .
$$

Since $\left\{\epsilon_{k}\right\}_{k=1}^{\infty}$ decreases to zero, then for any $\tau>0$

$$
\begin{aligned}
F_{f(\xi) f\left(\xi^{\prime}\right)}^{*}(\tau) & =\limsup _{n \rightarrow \infty} F_{f(\xi) f\left(\xi^{\prime}\right)}^{n}(\tau) \\
& \geq \limsup _{r \rightarrow \infty} F_{f(\xi) f\left(\xi^{\prime}\right)}^{N_{k_{r}}}(\tau) \\
& \geq \lim _{r \rightarrow \infty} \frac{N_{k_{r}}-N_{k_{r}}^{\prime}+1}{N_{k_{r}}} \\
& \geq \lim _{r \rightarrow \infty} \frac{k_{r}-1}{k_{r}}=1 .
\end{aligned}
$$

Therefore, $\left\{f(\xi), f\left(\xi^{\prime}\right)\right\}$ is a distributionally chaotic pair for any distinct points $\xi, \xi^{\prime} \in D$ and hence $f(D)$ is a distributionally $\epsilon$-scrambled set. This ends the proof.

In fact, we could extend it in some way. For instance, we have

Theorem 3.4 (Weakly Distributionally Chaotic Criterion). Let $X$ be a Banach space and let $T \in \mathcal{L}(X)$. If for any sequence of positive numbers $\left\{C_{m}\right\}_{m=1}^{\infty}$ increasing to $+\infty$ there exists a sequence of nontrivial points $\left\{x_{m}\right\}_{m=1}^{\infty}$ in $X$ satisfying

(WNU1) $\quad \lim _{k \rightarrow \infty}\left\|T^{k} x_{m}\right\|=0$, and

(WNU2) there is a sequence of positive integers $N_{m}$ increasing to $+\infty$, such that $\lim _{m \rightarrow \infty} \frac{\#\left\{0 \leq i \leq N_{m} ;\left\|T^{i} x_{m}\right\| \geq C_{m}\left\|x_{m}\right\|\right\}}{N_{m}}=1$,

then $T$ is distributionally chaotic.

The proof is similar to that of Theorem 3.3. According to Grosse-Erdmann's characterization [7, it is not difficult to see that Devaney chaotic backward shift operators satisfy the conditions of Theorem 3.4. So one can get the following conclusion immediately.

Corollary 3.5. If $T$ is a Devaney chaotic backward shift operator, then $T$ is distributionally chaotic.

Remark 3.6. This result is contained in a recent article [6], in which F. MartínezGiménez, P. Oprocha and A. Peris considered distributional chaos for shift operators. 
Applying this distributionally chaotic criterion (Theorem 3.3), we could provide a sufficient condition which gives distributional chaos for Cowen-Douglas operators.

Theorem 3.7. Let $T \in \mathcal{B}_{n}(\Omega)$. If $\Omega \cap S \neq \phi$, then $T$ is special. Consequently, $T$ is distributionally chaotic.

Proof. Since $\Omega$ is a connected open subset and $\Omega \cap S \neq \phi$, there exists $\beta \in \Omega$ with $|\beta|>1$. Furthermore, we can select a nontrivial point $y \in \operatorname{ker}(T-\beta)$. Let $1<$ $\gamma<|\beta|$ be a constant. Given any $m \in \mathbb{N}$, set $\epsilon<\frac{\|y\|}{2} \cdot \min \left\{1, \frac{|\beta|^{i}-\gamma^{i}}{|\beta|^{i}+1}, 1 \leq i \leq m\right\}$. Then $U=\bigcap_{i=0}^{m} T^{-i}\left(B\left(T^{i} y, \epsilon\right)\right)$ is an open neighborhood of $y$. Then for any $z \in U$,

$\left\|T^{i} z\right\| \geq\left\|T^{i} y\right\|-\epsilon=|\beta|^{i}\|y\|-\epsilon \geq|\beta|^{i}\|z\|-\left(|\beta|^{i}+1\right) \epsilon \geq \gamma^{i}\|z\|, i=1, \ldots, m$.

By hypothesis, one can obtain a bounded connected open subset $\Omega_{1}$ such that

$$
\sup _{\alpha \in \Omega_{1}}|\alpha|=\lambda<1 \text {. }
$$

By Proposition 1.5, there exists a point $x \in U$ satisfying

$$
x=\sum_{j=1}^{t} x_{j},
$$

where $x_{j} \in \operatorname{ker}\left(T-\lambda_{j}\right), \lambda_{j} \in \Omega_{1}, j=1, \ldots, t$. Then this $x$ is the point we hope to get. On the one hand,

$$
\lim _{k \rightarrow \infty}\left\|T^{k} x\right\|=\lim _{k \rightarrow \infty}\left\|\sum_{j=1}^{t} \lambda_{j}^{k} x_{j}\right\| \leq \lim _{k \rightarrow \infty} \sum_{j=1}^{t}\left|\lambda_{j}^{k}\right| \cdot\left\|x_{j}\right\| \leq\left(\sum_{j=1}^{t}\left\|x_{j}\right\|\right) \lim _{k \rightarrow \infty} \lambda^{k}=0 .
$$

On the other hand, according to the previous statement and $x \in U$, we have

$$
\left\|T^{i} x\right\| \geq \gamma^{i}\|x\|, \quad i=1,2, \ldots, m .
$$

Therefore $T$ is special, and hence $T$ is distributionally chaotic by Theorem 3.3 .

Example 3.8. Let $2 B$ be the backward shift operator with constant weight 2 . Then $2 B$ is special by Theorem 3.7. Moreover, for every $\lambda \in \mathbb{C}$ with $|\lambda|<3$, $\lambda I+2 B$ is also special since $\lambda I+2 B$ is in $\mathcal{B}_{1}(\Omega)$ and $\Omega \cap S \neq \phi$. However, if $|\lambda|>3$, then $\lambda I+2 B$ is not a special operator. In fact, it is not Li-Yorke chaotic. Because $\sigma(\lambda I+2 B)$ is disjoint with the unit closed disk and consequently for each point $x$ except $0,\left\|(\lambda I+2 B)^{n} x\right\| \rightarrow \infty$ as $n \rightarrow \infty$.

\section{REFERENCES}

[1] J. Banks, J. Brooks, G. Cairns, G. Davis and P. Stacey, On Devaney's definition of chaos, Amer. Math. Monthly, Vol. 99, 1992, 332-334. MR1157223 (93d:54059)

[2] M. J. Cowen and R. G. Douglas, Complex geometry and operator theory, Acta Mathematica, Vol. 141(1), 1978, 187-261. MR501368 (80f:47012)

[3] R. L. Devaney, An introduction to chaotic dynamical systems, Benjamin/Cummings, 1986. MR811850 (87e:58142)

[4] J. Duan, X. C. Fu, P. D. Liu and A. Manning, A linear chaotic quantum harmonic oscillator, Appl. Math. Lett. 12(1), 1999, 15-19. MR1663405(99h:81054)

[5] X. C. Fu and J. Duan, Infinite-dimensional linear dynamical systems with chaoticity, J. Nonlinear Sci. 9(2), 1999, 197-211. MR1674380(2000a:37003)

[6] F. Martínez-Giménez, P. Oprocha and A. Peris, Distributional chaos for backward shifts, Journal of Mathematical Analysis and Applications, Vol. 351(2), 2009, 607-615. MR2473967

[7] K. G. Grosse-Erdmann, Hypercyclic and chaotic weighted shifts, Studia Math., Vol. 139(1), 2000, 47-68. MR 1763044 (2001f:47051) 
[8] W. Huang and X. Ye, Devaney chaos or 2-scattering implies Li-Yorke chaos, Topology Appl., Vol. 117, 2002, 259-272. MR.1874089 (2003b:37017)

[9] T. Y. Li and J. A. Yorke, Period three implies chaos, Amer. Math. Monthly 82(10), 1975, 985-992. MR0385028 (52:5898)

[10] Gongfu Liao, Lidong Wang and Xiaodong Duan, A chaotic function with a distributively scrambled set of full Lebesgue measure, Nonlinear Analysis: Theory, Methods and Applications, Vol. 66(10), 2007, 2274-2280. MR2311031(2008c:37020)

[11] Gongfu Liao, Zhenyan Chu and Qinjie Fan, Relations between mixing and distributional chaos, Chaos, Solitons and Fractals, in press, corrected proof, available online 16 September 2008.

[12] J. Mai, Devaney chaos implies existence of s-scrambled sets, Proc. Amer. Math. Soc. 132(9), 2004, 2761-2767. MR2054803 (2005a:37022)

[13] P. Oprocha, A quantum harmonic oscillator and strong chaos, J. Phys. A 39(47), 2006, 14559-14565. MR2277068 (2007i:81099)

[14] B. Schweizer and J. Smítal, Measures of chaos and a spectral decomposition of dynamical systems on the interval, Trans. Amer. Math. Soc. 344(2), 1994, 737-754. MR1227094 (94k:58091)

[15] S. Silverman, On maps with dense orbits and the definition of chaos, Rocky Mountain Jour. Math., Vol. 22, 1992, 353-375. MR1159963 (93j:54033)

[16] J. Smítal and M. Štefánková, Distributional chaos for triangular maps, Chaos, Solitons and Fractals, Volume 21, Issue 5, 2004, 1125-1128. MR2047330 (2005a:37017)

[17] F. Balibrea, J. Smítal and M. Štefánková, The three versions of distributional chaos, Chaos, Solitons and Fractals, Volume 23, Issue 5, 2005, 1581-1583. MR2101573 (2005f:37040)

[18] Hui Wang, Gongfu Liao and Qinjie Fan, Substitution systems and the three versions of distributional chaos, Topology and its Applications, Volume 156, Issue 2, 2008, 262-267. MR2475113

Department of Mathematics, Jilin University, 130012, Changchun, People's Republic OF CHINA

E-mail address: houbz@jlu.edu.cn

Department of Mathematics, Dalian University of Technology, 116024, Dalian, PeoPLE'S REPUBLIC OF ChINA

E-mail address: cuipuyu1234@sina.com.cn

Department of Mathematics, Jilin University, 130012, Changchun, People's Republic OF CHINA

E-mail address: caoyang@jlu.edu.cn 